TRANSACTIONS OF THE

AMERICAN MATHEMATICAL SOCIETY

Volume 358, Number 2, Pages 573-590

S 0002-9947(05)03816-X

Article electronically published on June 21, 2005

\title{
MACNEILLE COMPLETIONS AND CANONICAL EXTENSIONS
}

\author{
MAI GEHRKE, JOHN HARDING, AND YDE VENEMA
}

\begin{abstract}
Let $V$ be a variety of monotone bounded lattice expansions, that is, bounded lattices endowed with additional operations, each of which is order preserving or reversing in each coordinate. We prove that if $V$ is closed under MacNeille completions, then it is also closed under canonical extensions. As a corollary we show that in the case of Boolean algebras with operators, any such variety $V$ is generated by an elementary class of relational structures.

Our main technical construction reveals that the canonical extension of a monotone bounded lattice expansion can be embedded in the MacNeille completion of any sufficiently saturated elementary extension of the original structure.
\end{abstract}

\section{INTRODUCTION}

Given a lattice $L$ with additional operations, one frequently wishes to find a completion of $L$, and an extension of the additional operations to this completion, so that the resulting structure preserves some given set of identities satisfied by the original.

The first example of this is Dedekind's famous construction of the reals from the rationals. Not only did Dedekind define the real numbers as cuts of the rationals, but he also extended the basic arithmetic operations on the rationals to the reals, and showed these extensions preserved the usual identities of arithmetic. MacNeille 26] generalized Dedekind's construction to provide a method of completion for arbitrary posets. Here this completion will be called the MacNeille completion, although it is also known as the normal completion, and the completion by cuts.

The second type of completion of interest here, the canonical extension, was first introduced in the setting of Boolean algebras by Jónsson and Tarski [22]. This completion arises essentially from Stone's duality theorem, with the canonical completion of a Boolean algebra $B$ being the embedding of $B$ into the power set of its Stone space. Canonical extensions for bounded distributive lattices can be realized in a similar way either through the Stone space, or the Priestley space, of

Received by the editors January 28, 2004.

2000 Mathematics Subject Classification. Primary 06B23, 03G10; Secondary 03B45, 03C05, 03G25, 06E25.

Key words and phrases. MacNeille completion, canonical extension, lattices, lattice ordered algebras, Boolean algebra with operators.

The authors express their gratitude to the anonymous referee for carefully reading and commenting on the manuscript, and, in particular, for making a valuable suggestion. Thanks are also due to Tadeusz Litak and Rob Goldblatt for comments on earlier versions of this paper. The first author's research was partially supported by grant NSF01-4-21760 of the USA National Science Foundation. 
the distributive lattice. It is probably fair to say that such a study in the case of general bounded lattices was initiated by Gehrke \& Harding [9].

The matter of extending additional operations on a Boolean algebra to operations on its canonical extension was initiated by Jónsson \& Tarski [22, 23]. Although they were originally motivated by their study of a rather specific class, viz., relation algebras, their work includes preservation results for the validity of identities and quasi-identities on Boolean algebras with operators (BAOs). The later introduction by Kripke and others of relational semantics for modal logics further increased the activity in this area. See Goldblatt [19] for a historical overview of these developments. The key fact is that the closure of a variety of modal algebras under canonical extensions implies the completeness of the corresponding logic with respect to its Kripke semantics. Referring the reader to Blackburn, de Rijke \& Venema [3] for a textbook exposition of the field, we here confine ourselves to mentioning one culminating result to come out of the investigations in modal logic: Sahlqvist's theorem [29]. Translated into algebraic terminology, this result gives sufficient syntactic conditions for a BAO identity to be preserved under taking canonical extensions. It was later generalized to various kinds of lattice expansions; see for instance Gehrke, Nagahashi \& Venema 11 for a result in the setting of distributive lattices.

The corresponding issue of extending additional operations to the MacNeille completion seems to have been addressed mostly in a piecemeal fashion, with various authors noting that the varieties of Boolean algebras, Heyting algebras, closure algebras, and ortholattices [2, 25], among others, can naturally be considered to be closed under MacNeille completions via some natural extension of the operations. The first systematic study of extensions of additional operations to MacNeille completions was done in the setting of Boolean algebras with additional operations by Monk. His [27] contains some general preservation results concerning the validity of equations, but it also gives a negative result, viz., that in general, one needs complete additivity of the original operators to guarantee additivity of their extensions. Monk's work was taken up by Givant \& Venema 13, who gave a type of Sahlqvist theorem for preservation of identities under MacNeille completions, again in the setting of Boolean algebras with additional well-behaved operations.

The identities that Givant \& Venema showed are preserved under MacNeille completions form a subset of those known to be preserved under canonical extensions. This naturally brings up the question, also raised by Goldblatt [17, whether the closure of a variety of BAOs under MacNeille completions implies its closure under canonical extensions. In this paper we answer Goldblatt's question in the affirmative. Also, rather than restricting ourselves to varieties of BAOs, we work in the setting of monotone bounded lattice expansions, that is, bounded lattices with additional operations that preserve or reverse order in each coordinate. Our main result states that every variety of monotone bounded lattice expansions that is closed under MacNeille completions is also closed under canonical extensions. This more general setting encompasses the algebraic counterparts of many logics, and includes many lattice-ordered structures studied by algebraists. It is our purely algebraic treatment, rather than relational methods usually employed in studies of BAOs, that allows this greater level of generality. The possibility of further generalization of this result, perhaps to the setting of partially ordered sets with additional operations, remains a matter of future study. 
Our method of proof is to show, for any bounded lattice $L$ with additional monotone operations, that the canonical extension of $L$ can be embedded into the MacNeille completion of a saturated non-standard extension of $L$. That this is reminiscent of a well-known proof method and resulting theorem in modal logic (see Fine [7] and Goldblatt [15]) is not a coincidence - the result in modal logic essentially follows by applying ours to the special case of a complete atomic Boolean algebra with operations that are completely additive in each coordinate.

Another connection to the theory of Boolean algebras with operators concerns the relation between varieties of BAOs that are closed under taking canonical extensions on the one hand, and classes of relational structures that are closed under taking ultraproducts, on the other. Prime examples of the latter kind are provided by elementary classes, that is, those classes of relational structures that are definable by a set of formulas in first order logic. It is well known that one can construct a BAO from the power set of a relational structure, the so-called complex algebra of the structure. Doing so for all algebras in a class of relational structures, we may subsequently consider the variety generated by all of these complex algebras. Goldblatt [15] has strengthened the original result of Fine [7] to show that if a class of relational structures is closed under taking ultraproducts, then the variety of BAOs generated by the complex algebras of structures in this class is canonical, that is, closed under taking canonical extensions.

For a long time it was an open problem whether the converse of this results holds as well, and in particular, whether every canonical variety of BAOs is generated by some elementary class of relational structures. A recent result of Goldblatt, Hodkinson \& Venema [18 shows this is not the case in general. However, our results do imply that in the case of a variety of BAOs that is closed under taking MacNeille completions, the answer is positive: all such varieties are generated by elementary classes of structures. These results are discussed in detail in a separate section at the end of the paper.

Before closing this introduction we should briefly remark on a third method of completion that is often used, the ideal lattice completion. Roughly, the ideal lattice completion works well when the additional operations are order preserving, but is of limited use when order inverting operations are allowed. While our results show that canonical extensions are preferable to MacNeille completions in regards to preservation of identities, there is no such comparison possible between the ideal lattice completion and either the MacNeille or canonical completion. For example, the variety of Boolean algebras is closed under MacNeille completions but not under ideal lattice completions, and the variety of modular lattices is closed under ideal lattice completions (see Crawley \& Dilworth [6] ) but not under canonical extensions; see Harding [20].

\section{Preliminaries}

Throughout, all lattices are bounded. In this section we review the two kinds of lattice completions, and for each completion, we explain the two natural ways in which additional monotone operations on the lattice can be extended to these completions. We finish this section with a brief discussion of our approach to the notion of saturation.

Lattice completions. We find it convenient to work with abstract characterizations of the canonical extension and MacNeille completion. The characterization of 
the MacNeille completion below is due to Banaschewski [1] and independently to Schmidt [30], and that of the canonical extension is due to Gehrke and Harding [9].

Definition 2.1. Let $L$ be a lattice, let $C$ be a complete lattice, and let $L \leq C$. We say

(1) $L$ is join-dense in $C$ if each element of $C$ is a join of elements of $L$.

(2) $L$ is meet-dense in $C$ if each element of $C$ is a meet of elements of $L$.

(3) $L$ is dense in $C$ if each element of $C$ is both a join of meets and a meet of joins of elements of $L$.

(4) $L$ is compact in $C$ if for each $A, B \subseteq L$ with $\bigwedge A \leq \bigvee B$ there are finite $A^{\prime} \subseteq A, B^{\prime} \subseteq B$ with $\bigwedge A^{\prime} \leq \bigvee B^{\prime}$

Theorem 2.2. For any lattice $L$ there exists a complete lattice $C$ such that

(1) $L \leq C$.

(2) $L$ is both join-dense and meet-dense in $C$.

Further, if $C^{\prime}$ is another complete lattice satisfying (1) and (2), then there is a unique isomorphism from $C$ to $C^{\prime}$ fixing $L$. We call such $C$ the MacNeille completion of $L$ and denote it $\bar{L}$.

Theorem 2.3. For any lattice $L$ there exists a complete lattice $C$ such that

(1) $L \leq C$.

(2) $L$ is dense in $C$.

(3) $L$ is compact in $C$.

Further, if $C^{\prime}$ is another complete lattice satisfying (1), (2) and (3), then there is a unique isomorphism from $C$ to $C^{\prime}$ fixing $L$. We call such $C$ the canonical extension of $L$ and denote it $L^{\sigma}$.

Definition 2.4. Define the sets $K\left(L^{\sigma}\right)$ and $O\left(L^{\sigma}\right)$ of closed and open elements of $L^{\sigma}$ by

(1) $K\left(L^{\sigma}\right)$ is all elements of $L^{\sigma}$ that are meets of elements of $L$.

(2) $O\left(L^{\sigma}\right)$ is all elements of $L^{\sigma}$ that are joins of elements of $L$.

Remark 2.5. Note that density of $L$ in $L^{\sigma}$ means that each element of $L^{\sigma}$ is a join of closed elements and a meet of open elements. Also, $L$ being compact in $L^{\sigma}$ means that if a closed element $x$ lies beneath an open element $y$, then there is some element $a \in L$ with $x \leq a \leq y$. In particular, the elements of $L$ are exactly the clopen elements.

Remark 2.6. Almost all of our methods and results could be phrased in terms of various topologies that naturally arise in the context that we have just defined. We have decided to present the results in a topology-free environment. However, since we do believe the topological perspective to be very useful, we will occasionally point out a topological connection. For that purpose, we here introduce the three topological families that are involved.

To start with, there are the well-known lower, upper and interval topologies, which can in fact be defined on arbitrary partial orders. Given a poset $(P, \leq)$, obtain the lower topology $\iota^{\downarrow}$ and the upper topology $\iota^{\uparrow}$ on $P$ by taking as a subbasis the collection consisting of the empty set together with the collection of complements $P \backslash(\uparrow p)$ of principal upsets and the collection of complements $P \backslash(\downarrow p)$ of principal downsets, respectively. The interval topology $\iota$ is defined as the join of $\iota^{\downarrow}$ and $\iota^{\uparrow}$; its name is explained by the fact that it is the smallest topology for which all intervals 
$[p, q]=\{x \in P \mid p \leq x \leq q\}$, where $p$ and $q$ are either elements of $P$ or $+\infty$ or $-\infty$, are closed. In the case that $P$ is a complete lattice satisfying some (infinitary) distributive laws, these three topologies have various other nice characterizations as well; we need not go into the details here, referring to Gierz et al. [12] instead.

Perhaps the most important contribution of Gehrke and Jónsson [10] is the topological perspective on canonical extensions. They introduce on $L^{\sigma}$ the topologies $\sigma, \sigma^{\uparrow}$ and $\sigma^{\downarrow}$, having as their bases, respectively, the collections of sets of the form $[x, y], \uparrow x$ and $\downarrow y$, with $x \in K\left(L^{\sigma}\right)$ and $y \in O\left(L^{\sigma}\right)$. Using the denseness and compactness of $L$ in $L^{\sigma}$ one can show that the set $L$ is (topologically) dense in $\left(L^{\sigma}, \sigma\right)$, and that the members of $L$ are exactly the isolated points of $\left(L^{\sigma}, \sigma\right)$.

Similarly, on the MacNeille completion $\bar{L}$ of a lattice $L$, one can define topologies $\rho, \rho^{\uparrow}$ and $\rho^{\downarrow}$ generated by, respectively, the collection of all sets of the form $[a, b]$, $\uparrow a$ and $\downarrow b$, with $a, b \in L$. It is now easy to prove that $L$ is (topologically) dense in $\bar{L}$, and that the members of $L$ are exactly the isolated points of $(\bar{L}, \rho)$.

Extending monotone maps to lattice completions. We now explain the two natural ways in which additional, monotone operations on a lattice can be extended to the MacNeille completion and canonical extension. We begin by considering extensions of order preserving maps between lattices.

Definition 2.7. For $f: L \rightarrow M$ order preserving, define $\bar{f}, \widehat{f}: \bar{L} \rightarrow \bar{M}$ by

$$
\begin{aligned}
& \bar{f}(u)=\bigvee\{f(a) \mid a \leq u \text { and } a \in L\}, \\
& \widehat{f}(u)=\bigwedge\{f(a) \mid u \leq a \text { and } a \in L\} .
\end{aligned}
$$

We call $\bar{f}$ and $\widehat{f}$ the lower and upper MacNeille extensions of $f$, respectively.

In the case of a Boolean algebra $B$ with completely additive operators, the expansion of $\bar{B}$ with the lower extension of the operators is known in the literature as the (Monk) completion of the original structure; cf. [27, 21].

Definition 2.8. For $f: L \rightarrow M$ order preserving, define $f^{\sigma}, f^{\pi}: L^{\sigma} \rightarrow M^{\sigma}$ by

$$
\begin{aligned}
& f^{\sigma}(u)=\bigvee\left\{\bigwedge\{f(a) \mid x \leq a \in L\} \mid u \geq x \in K\left(L^{\sigma}\right)\right\}, \\
& f^{\pi}(u)=\bigwedge\left\{\bigvee\{f(a) \mid y \geq a \in L\} \mid u \leq y \in O\left(L^{\sigma}\right)\right\} .
\end{aligned}
$$

We call $f^{\sigma}$ and $f^{\pi}$ the lower and upper canonical extensions of $f$, respectively.

We shall next consider extensions of monotone maps, that is, maps of the form $f: L^{n} \rightarrow M$ that preserve or reverse the order in each coordinate. It will be convenient to introduce some terminology.

Definition 2.9. An element $\epsilon \in\{1, d\}^{n}$ will be called an order type or monotonicity type (of length $n$ ). Given a lattice $L$, we let $L^{\epsilon}$ denote the lattice product $\prod_{i=1}^{n} L^{\epsilon_{i}}$, where $L^{\epsilon_{i}}$ is $L$ if $\epsilon_{i}=1$, and $L^{\epsilon_{i}}$ is the order dual $L^{d}$ of $L$ if $\epsilon_{i}=d$.

Note that $L^{n}$ and $L^{\epsilon}$ are based on the same carrier set, so that a map $f: L^{n} \rightarrow M$ can (set-theoretically) be viewed as a map from $L^{\epsilon} \rightarrow M$ as well.

Definition 2.10 (Monotone lattice expansions). Let $\epsilon$ be a monotonicity type. An operation $f: L^{n} \rightarrow M$ is $\epsilon$-monotone if it is order preserving when viewed as a map from $L^{\epsilon} \rightarrow M$. An operation $f: L^{n} \rightarrow M$ is called monotone if it is $\epsilon$-monotone for some monotonicity type $\epsilon$. 
A monotone lattice expansion is a bounded lattice expanded with a family of monotone operations.

Definition 2.11. Let $f: L^{n} \rightarrow L$ be a monotone operation of type $\epsilon$. Viewing $f$ as an order preserving map from $L^{\epsilon}$ to $L$, we let $\bar{f}$ and $\widehat{f}$, respectively, denote the lower and upper MacNeille extension of $f$, as in Definition 2.7 Similarly, we define the lower and upper canonical extensions $f^{\sigma}$ and $f^{\pi}$ of $f$, as in Definition 2.8.

The reader may worry that the above definition depends on the monotonicity type of $f$. So what if there would not be a unique $\epsilon \in\{1, d\}^{n}$ such that $f$ is $\epsilon$ monotone? Fortunately, this could only happen if $f$ is both order preserving and reversing in some coordinates - but then $f$ does not depend on the argument in those coordinates. This shows that $\bar{f}, \widehat{f}, f^{\sigma}$ and $f^{\pi}$ have been well defined.

Using the facts that $\overline{\left(L^{\epsilon}\right)}=(\bar{L})^{\epsilon}$ and $\left(L^{\epsilon}\right)^{\sigma}=\left(L^{\sigma}\right)^{\epsilon}$, it is easy to find more direct definitions of the extensions of monotone maps.

Example 2.12. A Heyting algebra $H$ is a bounded distributive lattice with an additional binary operation $f$ (often written $\rightarrow$ ) satisfying certain identities. The operation $f$ is order reversing in the first coordinate and order preserving in the second. Then

$$
\begin{aligned}
& \bar{f}(u, v)=\bigvee\{f(a, b) \mid u \leq a, b \leq v \text { and } a, b \in H\}, \\
& \widehat{f}(u, v)=\bigwedge\{f(a, b) \mid a \leq u, v \leq b \text { and } a, b \in H\} .
\end{aligned}
$$

Also, as $K\left(\left(H^{d} \times H\right)^{\sigma}\right)=O\left(H^{\sigma}\right) \times K\left(H^{\sigma}\right)$ and $O\left(\left(H^{d} \times H\right)^{\sigma}\right)=K\left(H^{\sigma}\right) \times O\left(H^{\sigma}\right)$, we have

$$
\begin{aligned}
& f^{\sigma}(u, v)=\bigvee\left\{\bigwedge\{f(a, b) \mid x \geq a, y \leq b\} \mid u \leq x \in O\left(H^{\sigma}\right), v \geq y \in K\left(H^{\sigma}\right)\right\}, \\
& f^{\pi}(u, v)=\bigwedge\left\{\bigvee\{f(a, b) \mid x \leq a, b \leq y\} \mid u \geq x \in K\left(H^{\sigma}\right), v \leq y \in O\left(H^{\sigma}\right)\right\} .
\end{aligned}
$$

It is well known that the lower completions $(\bar{H}, \bar{f})$ and $\left(H^{\sigma}, f^{\sigma}\right)$ need not be Heyting algebras, while the upper completions $(\bar{H}, \widehat{f})$ and $\left(H^{\sigma}, f^{\pi}\right)$ are Heyting algebras.

Remark 2.13. There is a natural way to generalize the above definitions to apply to extensions of arbitrary maps between lattices, and in particular, to extensions of arbitrary operations on a lattice. This technique was established for canonical extensions by Gehrke \& Jónsson [10]. For instance, to define the lower extension $f^{\sigma}$ for an arbitrary map $f: L \rightarrow M$, one can take

$$
f^{\sigma}(u)=\bigvee\left\{\bigwedge\left\{f(a) \mid a \in[x, y]_{L}\right\} \mid x \leq u \leq y, x \in K\left(L^{\sigma}\right), y \in O\left(L^{\sigma}\right)\right\},
$$

where $[x, y]_{L}$ denotes the set $\{a \in L \mid x \leq a \leq y\}$.

From the perspective of the topologies introduced in Remark 2.6, this definition can be seen as

$$
f^{\sigma}=\underline{\lim }_{\sigma} f,
$$

where, for a dense subset $D$ of a topological space $(X, \tau)$, a complete lattice $C$ and a map $g: D \rightarrow C$, one puts

$$
\varliminf_{\tau} g(u)=\bigvee\{\bigwedge g(U \cap D) \mid u \in U \in \tau\} .
$$

The fact that every element of $L$ is an isolated point of $\left(L^{\sigma}, \sigma\right)$ then implies that $f^{\sigma}$ is indeed an extension of $f$. Connecting the topological families, $\left\{\sigma^{\uparrow}, \sigma^{\downarrow}, \sigma\right\}$ on $L^{\sigma}$ and $\left\{\iota^{\uparrow}, \iota^{\downarrow}, \iota\right\}$ on $M^{\sigma}$, one can show that the maps $f^{\sigma}$ and $f^{\pi}$ are the largest 
$\left(\sigma, \iota^{\uparrow}\right)$-continuous and the smallest $\left(\sigma, \iota^{\downarrow}\right)$-continuous extension of $f$, respectively. Likewise, involving the $\rho$ topologies on the MacNeille completion of the domain, it holds that $\bar{f}$ and $\widehat{f}$ are the largest $\left(\rho, \iota^{\uparrow}\right)$-continuous and the smallest $\left(\rho, \iota^{\downarrow}\right)$ continuous extension of $f$, respectively.

Many useful properties of maps between lattices can be expressed in terms of these topologies. To mention a couple of examples, it follows almost immediately from the facts mentioned above that the map $f: L \rightarrow M$ is smooth (meaning that its lower and upper extension coincide: $f^{\sigma}=f^{\pi}$ ) if and only if $f$ has a $(\sigma, \iota)$ continuous extension $g: L^{\sigma} \rightarrow M^{\sigma}$. Other useful consequences of the topological characterizations concern the fact that in some cases, the operation of extending maps commutes with that of composing them. For example, if $g^{\sigma}$ happens to be $(\sigma, \sigma)$-continuous, then $f^{\sigma} g^{\sigma} \leq(f g)^{\sigma}$. This latter statement is actually of the kind involved in our proofs. For more details concerning this approach to canonical extensions the reader is referred to Gehrke \& Jónsson [10].

While the assumption of monotonicity can be avoided in the definition of the extensions of additional operations, the assumption of monotonicity is used in the proof of several of our key results. For this reason we will remain in the setting of monotone lattice expansions throughout this paper.

Saturated extensions. Finally, we shall use results from non-standard analysis extensively. The reader should consult Gehrke [8] for an introduction to the use of non-standard analysis in lattice theory in particular, and Stroyan and Luxemburg 31 for details on non-standard analysis in general.

In brief, a binary relation $R$ is concurrent on a subset $X$ of its domain if for any finite number $n$ of elements $x_{1}, \ldots, x_{n}$ in $X$ there is some $y$ with $x_{1} R y, \ldots, x_{n} R y$. A non-standard extension $L^{*}$ of $L$ is called $\kappa$-saturated if for any internal binary relation $R$ of $L^{*}$ that is concurrent on a subset $X$ of its domain with cardinality less than $\kappa$, there is a $y$ with $x R y$ for all $x \in X$. We remark that $R$ need not be a relation over the individuals of $L^{*}$, but could, for instance, be an internal binary relation over the $n$-fold power of the individuals of $L^{*}$. This fact will be of importance in later considerations of $n$-ary operations.

The crucial point is that for any structure $L$ and any cardinal $\kappa$, there exists a $\kappa$-saturated non-standard extension of $L$. In fact, such a $\kappa$-saturated extension of $L$ can be chosen to be an ultrapower of $L$. For a complete account, see 31 .

For readers who are more familiar with the concept of saturation from classical model theory, rather than non-standard analysis, it will not be difficult to replace the details of our proofs with an argumentation based on notions from Chang \& Keisler [5]. At the end of section 3 we provide a sketch of this alternative approach.

\section{MAIN RESULTS}

Throughout $L$ is a monotone lattice expansion of cardinality $\kappa$ and $L^{*}$ is a $\kappa^{+}$saturated non-standard extension of $L$. Here $\kappa^{+}$is the successor cardinal to $\kappa$. We first show that there is a complete lattice embedding of the canonical extension of $L$ into the MacNeille completion of $L^{*}$.

Definition 3.1. For $i: L \rightarrow \overline{L^{*}}$ the identity map, define $\varphi: L^{\sigma} \rightarrow \overline{L^{*}}$ by

$$
\varphi(u)=\bigvee\left\{\bigwedge\{i(a) \mid x \leq a \in L\} \mid u \geq x \in K\left(L^{\sigma}\right)\right\} .
$$


Since $i(a)=a$ for all $a \in L$, it makes no difference whether we denote an element of $L$ as $a$ or $i(a)$. In the sequel we will usually choose the first option, writing $i(a)$ only if we feel it helps the reader to focus on where things take place.

Theorem 3.2. The map $\varphi$ is the unique complete lattice embedding of $L^{\sigma}$ into $\overline{L^{*}}$ that extends the identical embedding.

Proof. It is not difficult to see that $\varphi$ is an order preserving extension of the identity embedding $i$. In order to prove the other properties of $\varphi$, we need the following technical fact:

For all $p \in L^{*}$ and $y \in O\left(L^{\sigma}\right)$, if $p \leq \varphi(y)$, then there is some $a \in L$ with $a \leq y$ and $p \leq i(a)$.

The condition (3.1) can be understood both lattice theoretically and topologically. Lattice theoretically, one can show that (3.1) is equivalent to saying that any ideal $I$ of $L$ generates a normal ideal of $L^{*}$. For the topological interpretation, one can show that the condition as stated is equivalent to the following condition:

For all $p \in L^{*}$ and $u \in L^{\sigma}$, if $p \leq \varphi(u)$, then there is some $x \in K\left(L^{\sigma}\right)$ with

$x \leq u$ and $p \leq \varphi(x)$,

which one may easily verify to spell out the condition of $\varphi$ being $\left(\sigma^{\uparrow}, \rho^{\uparrow}\right)$-continuous.

To prove (3.1), pick $p \in L^{*}, y \in O\left(L^{\sigma}\right)$ with $p \leq \varphi(y)$, and set $I=\{a \in L \mid a \leq y\}$. Suppose $p \not \leq a$ for each $a \in I$. Define a relation $R$ by setting $u R v \Leftrightarrow u \leq v$ and $p \not \leq v$. For any $a_{1}, \ldots, a_{n} \in I$ we have that $a=a_{1} \vee \cdots \vee a_{n}$ belongs to $I$, so by assumption $p \not \leq a$, therefore $a_{1} R a, \ldots, a_{n} R a$. So the internal binary relation $R$ is concurrent on the set $I$ of cardinality less than $\kappa^{+}$, and therefore there is $z \in L^{*}$ with $a R z$ for all $a \in I$. Thus $p \not \leq z$ and $i(a)=a \leq z$ for all $a \in I$. By the compactness of $L$ in $L^{\sigma}$ we have $\varphi(y)=\bigvee\{i(a) \mid y \geq a \in L\}$, so $\varphi(y) \leq z$, contrary to the assumption that $p \leq \varphi(y)$. This proves (3.1).

We next turn to the proof that $\varphi$ is a complete lattice homomorphism, that is, preserves arbitrary meets and joins. We first show that $\varphi$ preserves joins (in $L^{\sigma}$ ) of elements of $L$. Suppose $a_{i}(i \in I)$ is a family of elements of $L$. As $\varphi$ is order preserving we have $\bigvee_{I} \varphi\left(a_{i}\right) \leq \varphi\left(\bigvee_{I} a_{i}\right)$. Setting $y=\bigvee_{I} a_{i}$, the compactness of $L$ in $L^{\sigma}$ gives $\varphi(y)=\bigvee\{i(b) \mid y \geq b \in L\}$. But if $b \leq y=\bigvee_{I} a_{i}$, then by compactness $b \leq a_{i_{1}} \vee \cdots \vee a_{i_{n}}$ for some $i_{1}, \ldots, i_{n}$. It follows that $\varphi\left(\bigvee_{I} a_{i}\right) \leq \bigvee_{I} \varphi\left(a_{i}\right)$.

We next show that $\varphi$ preserves arbitrary meets. Note that as each element of $L^{\sigma}$ is a meet of open elements, it is enough to show $\varphi\left(\bigwedge_{I} y_{i}\right)=\bigwedge_{I} \varphi\left(y_{i}\right)$ whenever $y_{i}(i \in I)$ is a family of open elements. That $\varphi\left(\bigwedge_{I} y_{i}\right) \leq \bigwedge_{I} \varphi\left(y_{i}\right)$ follows as $\varphi$ is order preserving. As $L^{*}$ is join dense in $\overline{L^{*}}$, for the other inequality it is enough to show each $p \in L^{*}$ under $\bigwedge_{I} \varphi\left(y_{i}\right)$ is also under $\varphi\left(\bigwedge_{I} y_{i}\right)$. By (3.1), as $p \leq \varphi\left(y_{i}\right)$, there is $a_{i} \leq y_{i}$ with $a_{i} \in L$ and $p \leq \varphi\left(a_{i}\right)$. Set $x=\bigwedge_{I} a_{i}$. Then $x \leq \bigwedge_{I} y_{i}$ and $\varphi(x)=\bigwedge\{i(c) \mid x \leq c \in L\}$. But if $x=\bigwedge_{I} a_{i} \leq c \in L$, we have by compactness $a_{i_{1}} \wedge \cdots \wedge a_{i_{n}} \leq c$ for some $i_{1}, \ldots, i_{n}$, hence $p \leq c$, so $p \leq \varphi(x) \leq \varphi\left(\bigwedge_{I} y_{i}\right)$ as required.

By duality the map $\psi: L^{\sigma} \rightarrow \overline{L^{*}}$ defined by

$$
\psi(u)=\bigwedge\left\{\bigvee\{i(a) \mid y \geq a \in L\} \mid u \leq y \in O\left(L^{\sigma}\right)\right\}
$$

is an extension of the identity that preserves arbitrary joins. But for $u \in L^{\sigma}$ we have $u=\bigwedge\left\{\bigvee\{a \mid y \geq a \in L\} \mid u \leq y \in O\left(L^{\sigma}\right)\right\}$. Then as $\varphi$ preserves arbitrary (including 
infinitary) joins of elements of $L$, and also arbitrary meets, we have $\varphi(u)=\psi(u)$. Thus $\varphi$ preserves arbitrary joins and meets.

In order to prove the injectivity of $\varphi$, we claim that it suffices to show the following:

$$
\text { If } \varphi(x) \leq \varphi(y) \text { for } x \in K\left(L^{\sigma}\right) \text { and } y \in O\left(L^{\sigma}\right) \text {, then } x \leq y \text {. }
$$

Suppose (3.2) holds and $u, v \in L^{\sigma}$ are such that $\varphi(u) \leq \varphi(v)$. Then $\varphi(x) \leq \varphi(y)$ for all closed $x \leq u$ and all open $y \geq v$ since $\phi$ preserves order. So, by (3.2), $x \leq y$ for each closed $x \leq u$ and open $y \geq v$, hence by density $u \leq v$. Thus $\varphi$ is an order embedding, and therefore is injective.

For the proof of (3.2), set $F=\{a \in L \mid x \leq a\}$ and define a relation $R$ by setting $u R v \Leftrightarrow u \geq v$ and $v \in F^{*}$. This relation is not only internal but actually standard. Also, for any $a_{1}, \ldots, a_{n} \in F$ we have that $a=a_{1} \wedge \cdots \wedge a_{n}$ belongs to $F$, so $a_{1} R a, \ldots, a_{n} R a$. Because $R$ is standard, $\kappa^{+}$-saturation is not even needed to conclude that there is $p \in F^{*}$ with $p \leq a=i(a)$ for all $a \in F$, as this is true in any non-standard extension; e.g. see [8]. Now, as $\varphi(x)=\bigwedge\{i(a) \mid x \leq a \in L\}$ we have $p \leq \varphi(x)$, hence as $\varphi(x) \leq \varphi(y)$, we have $p \leq \varphi(y)$. Condition (3.1) then yields $a \in L$ with $p \leq a$ and $a \leq y$. As $F$ is a filter of $L$, then $F^{*}$ is a filter of $L^{*}$. Then as $p \in F^{*}$ and $p \leq a$, we have $a \in F^{*}$, and as $a$ is standard, $a \in F$. Therefore $x \leq a \leq y$.

Finally, uniqueness of $\varphi$ follows as $L^{\sigma}$ is completely generated by $L$.

We now show that the embedding $\varphi$ is also a homomorphism with respect to the additional operations.

Proposition 3.3. Suppose $f$ is a monotone n-ary operation on $L$. Then for all $u_{1}, \ldots, u_{n} \in L^{\sigma}$ :

$$
\begin{aligned}
& \overline{f^{*}}\left(\varphi\left(u_{1}\right), \ldots, \varphi\left(u_{n}\right)\right)=\varphi f^{\sigma}\left(u_{1}, \ldots, u_{n}\right), \\
& \widehat{f^{*}}\left(\varphi\left(u_{1}\right), \ldots, \varphi\left(u_{n}\right)\right)=\varphi f^{\pi}\left(u_{1}, \ldots, u_{n}\right) .
\end{aligned}
$$

Proof. To avoid introducing cumbersome notation we prove the result in detail for the lower extension $\overline{f^{*}}$ of an order preserving unary operation and indicate the changes necessary for a binary operation that preserves order in the first coordinate and reverses order in the second coordinate. The argument for the lower extension of a general monotone operation is merely a formalization of this, and the argument for upper extensions follows by duality, as $\varphi$ is the unique extension of the identity map to a complete homomorphism.

Suppose $f: L \rightarrow L$ is order preserving. We need the following fact:

$$
\begin{aligned}
& \text { For all } p \in L^{*} \text { and } x \in K\left(L^{\sigma}\right) \text {, if } p \geq \overline{f^{*}} \varphi(x) \text {, then there is some } a \in L \\
& \text { with } a \geq x \text { and } p \geq i(f(a)) \text {. }
\end{aligned}
$$

Condition (3.3) is fairly transparently a continuity condition, namely:

For all $p \in L^{*}$ and $x \in K\left(L^{\sigma}\right)$, if $p \geq \overline{f^{*}} \varphi(x)$, then there is $y \in O\left(L^{\sigma}\right)$

with $y \geq x$ and $p \geq \overline{f^{*}} \varphi(y)$.

Condition (3.3) is obtained from this latter condition by applying compactness to the inequality $y \geq x$ and then applying the definitions of the various functions. But this latter condition is exactly the condition that $\overline{f^{*}} \varphi$ is $\left(\sigma^{\downarrow}, \rho^{\downarrow}\right)$-continuous on 
$K\left(L^{\sigma}\right)$. Now one may wonder whether, just as for condition (3.1), we have that this in turn is equivalent to:

For all $p \in L^{*}$ and $u \in L^{\sigma}$, if $p \geq \overline{f^{*}} \varphi(u)$, then there is some $y \in O\left(L^{\sigma}\right)$

with $y \geq u$ and $p \geq \overline{f^{*}} \varphi(y)$,

which is the condition that $\overline{f^{*}} \varphi$ is $\left(\sigma^{\downarrow}, \rho^{\downarrow}\right)$-continuous on all of $L^{\sigma}$. However, this is not the case at this level of generality. But, if $f$ is an operator (modulo the appropriate flipping of coordinates in the $n$-ary case), then it is true.

For the proof of (3.3), take $x \in K\left(L^{\sigma}\right), p \in L^{*}$, and let $F=\{a \in L \mid x \leq a\}$. Assume $f(a) \not \leq p$ for each $a \in F$. We must show $\overline{f^{*}} \varphi(x) \not \leq p$. Define a relation $R$ by setting $u R v \Leftrightarrow u \geq v$ and $f^{*}(v) \not \leq p$. For any $a_{1}, \ldots, a_{n} \in F$ if we set $a=a_{1} \wedge \cdots \wedge a_{n}$, then $a_{1} R a, \ldots, a_{n} R a$ since $f(a) \not \leq p$ for each $a \in F$. So the internal binary relation $R$ is concurrent on the set $F$ of cardinality less than $\kappa^{+}$, thus there is $z \in L^{*}$ with $a R z$ for all $a \in F$. This means that $z \leq a$ for all $a \in F$, hence $z \leq \varphi(x)$, and $f^{*}(z) \not p$. It then follows by the monotonicity of $\overline{f^{*}}$ that $\overline{f^{*}} \varphi(x) \not \leq p$. This proves (3.3).

We first show that $\overline{f *} \varphi(x)=\varphi f^{\sigma}(x)$ for $x$ closed. As $L^{*}$ is meet-dense in $\overline{L^{*}}$, condition (3.3) provides $\overline{f^{*}} \varphi(x) \geq \bigwedge\{\varphi f(a) \mid x \leq a \in L\}$ and monotonicity provides the other inequality. Then as $\varphi$ is complete, $\bar{f}^{*} \varphi(x)=\varphi f^{\sigma}(x)$.

For arbitrary $u \in L^{\sigma}$ we have $\varphi f^{\sigma}(u)=\varphi\left(\bigvee\left\{f^{\sigma}(x) \mid u \geq x \in K\left(L^{\sigma}\right)\right\}\right)$, and using the fact that $\varphi$ is complete, in conjunction with the above result for closed elements, $\varphi f^{\sigma}(u)=\bigvee\left\{\overline{f^{*}} \varphi(x) \mid u \geq x \in K\left(L^{\sigma}\right)\right\}$. Monotonicity then gives $\varphi f^{\sigma}(u) \leq \overline{f^{*}} \varphi(u)$. For the other inequality note $\overline{f^{*}} \varphi(u)=\bigvee\left\{f^{*}(p) \mid \varphi(u) \geq p \in L^{*}\right\}$ by definition of $\overline{f^{*}}$. Suppose $p \leq \varphi(u)$, where $p \in L^{*}$. Expressing $u$ as a meet of open elements $u=\bigwedge_{I} y_{i}$ we have $p \leq \varphi\left(y_{i}\right)$ for each $i \in I$. Then by (3.1), $p \leq \varphi\left(a_{i}\right)$ for some $a_{i} \leq y_{i}$. Setting $x=\bigwedge_{I} a_{i}$ we have that $x$ is closed, $x \leq u$, and as $\varphi$ is complete, $p \leq \varphi(x)$. It follows that $f^{*}(p) \leq \overline{f^{*}} \varphi(x)=\varphi f^{\sigma}(x)$, and so, by monotonicity, $f^{*}(p) \leq \varphi f^{\sigma}(u)$. Therefore $\overline{f^{*}} \varphi(u) \leq \varphi f^{\sigma}(u)$.

The argument for a binary operation preserving order in the first coordinate and reversing order in the second requires the statement of (3.3) to be modified to apply to closed elements $(x, y)$ of $\left(L \times L^{d}\right)^{\sigma}$. Specifically, for $x \in K\left(L^{\sigma}\right)$ and $y \in O\left(L^{\sigma}\right)$ with $\overline{f^{*}}(\varphi(x), \varphi(y)) \leq p$ we must show there are $a, b \in L$ with $x \leq a$, $b \leq y$ and $f(a, b) \leq p$. This is accomplished by considering the binary relation $R$ on $L^{*} \times L^{*}$ defined by $\left(u_{1}, u_{2}\right) R\left(v_{1}, v_{2}\right) \Leftrightarrow u_{1} \geq v_{1}, u_{2} \leq v_{2}$ and $f^{*}\left(v_{1}, v_{2}\right) \not \leq p$, and then noting that $R$ is concurrent on the set $F=\{(a, b) \in L \times L \mid x \leq a, b \leq y\}$. The argument then follows the lines above showing first that $\overline{f^{*}}(\varphi(x), \varphi(y))=\varphi f^{\sigma}(x, y)$ for $x$ closed and $y$ open, and then using this result and (3.1) and its dual to show equality for arbitrary $u, v \in L^{\sigma}$.

We now have all the ingredients for our main technical result, viz., Theorem 3.5 below, which extends Theorem 3.2 to monotone lattice expansions. In the most general formulation, we may be dealing with lattices endowed with more than one additional operation. Depending on whether we take the lower or upper extension for each of these, we arrive at a different extension of the algebra. We need some terminology for this.

Definition 3.4. Suppose $L$ is a lattice with a family of additional operations indexed by the set $I$. A completion type $\beta$ for $L$ is a specification of which operations $f_{i}$ are to be extended with the lower extension, and which are to be extended with 
the upper extension. The $\beta$-MacNeille and $\beta$-canonical completions of $L$ are then defined in the obvious ways.

Theorem 3.5. Suppose $L$ is a lattice of cardinality $\kappa$ expanded with a family of monotone operations. Then for any $\kappa^{+}$-saturated non-standard extension $L^{*}$ and any completion type $\beta$ of $L$, the $\beta$-canonical extension of $L$ can be embedded into the $\beta$-MacNeille completion of $L^{*}$ via an embedding that preserves all joins and meets.

Proof. Theorem 3.2 shows $\varphi$ is a complete embedding of the lattice $L^{\sigma}$ into $\overline{L^{*}}$. The previous proposition shows that $\varphi$ is compatible with the extensions of the operation $f_{i}$, provided both are lower extensions or both are upper extensions.

Theorem 3.5 paves the way for our main result which states that varieties that are closed under MacNeille completions are also closed under canonical extensions. In fact, we can prove something slightly stronger.

We recall a formula $\Phi$ is a universal formula if all the quantifiers of its prenex normal form are universal quantifiers. A class $K$ is a universal class if it can be axiomatized by universal formulas. It is well known (see for instance Burris \& Sankappanavar [4, pg. 215]) that a class $K$ is universal iff it is closed under isomorphism, substructures and ultraproducts.

Theorem 3.6. Let $K$ be a universal class (for instance, a variety) of monotone lattice expansions. If $K$ is closed under $\beta$-MacNeille completions, then $K$ is closed under $\beta$-canonical extensions.

Proof. Suppose $L$ belongs to $K$ and $L$ has cardinality $\kappa$. Let $L^{*}$ be a $\kappa^{+}$-saturated ultrapower of $L$. Then $L^{*}$ also belongs to $K$, and as the $\beta$-canonical extension of $L$ is isomorphic to a subalgebra of the $\beta$-MacNeille completion of $L^{*}$, the result follows.

The following lemma is required to extend Theorem 3.6 .

Lemma 3.7. Suppose $L_{i}(i \in I)$ is a family of monotone lattice expansions of the same type and $\beta$ is a completion type for this family. Then for any ultrafilter $\mu$ over $I$,

$$
\overline{\prod_{\mu} L_{i}} \simeq \overline{\prod_{\mu} \overline{L_{i}}}
$$

Here $\prod_{\mu} L_{i}$ is the ultraproduct of the $L_{i}$ and $\bar{L}$ is the $\beta$-MacNeille completion of $L$.

Proof. We establish this result for the case where the $L_{i}$ have a single binary operation that preserves order in the first coordinate and reverses order in the second, and where $\beta$ indicates taking the lower extension of this operation. Other cases are obviously similar.

Let $f_{i}$ be the additional operation on $L_{i}$ and for convenience let $g_{i}=\overline{f_{i}}$ be the lower extension of $f_{i}$ to $\overline{L_{i}}$. Let $(A, f)$ be the ultraproduct $\prod_{\mu}\left(L_{i}, f_{i}\right)$ and let $(B, g)$ be the ultraproduct $\prod_{\mu}\left(\overline{L_{i}}, g_{i}\right)$.

Extend our language to include a unary predicate $P_{i}$ on each $\overline{L_{i}}$, where $P_{i}(a)$ says that $a \in L_{i}$. Let $P$ be the induced predicate on the ultraproduct $\prod_{\mu} \overline{L_{i}}$, and note that up to a minor abuse of notation $P(a)$ says that $a$ is an element of $\prod_{\mu} L_{i}$. Using this predicate, we can then find first order statements $\varphi$ and $\psi$ whose interpretations in $\overline{L_{i}}$ say, respectively, that $L_{i}$ is join and meet dense in $\overline{L_{i}}$ and that 
for each $a, b \in \overline{L_{i}}$ we have $g_{i}(a, b)=\bigvee\left\{f_{i}(c, d) \mid c, d \in L_{i}\right.$ and $\left.c \leq a, b \leq d\right\}$. For instance, join-density is expressed by saying that for all $a, b$, if $a \not \leq b$, then there is some $c$ with $P(c)$ and $c \leq a$, but not $c \leq b$.

As $\varphi$ and $\psi$ hold in each $\overline{L_{i}}$, they also hold in the ultraproduct $(B, g)$. Thus, $A$ is join and meet dense in $B$ and $g(a, b)=\bigvee\{f(c, d) \mid c, d \in A$ and $c \leq a, b \leq d\}$ for each $a, b \in B$. Consider the lower MacNeille completion $\overline{(B, g)}=(\bar{B}, \bar{g})$ of $(B, g)$. As $A$ is join and meet dense in $B$, it follows that $A$ is join and meet dense in the complete lattice $\bar{B}$. Also, for any $x, y \in \bar{B}$ we have

$$
\bar{g}(x, y)=\bigvee\{g(a, b) \mid a, b \in B \text { and } a \leq x, y \leq b\},
$$

and it follows from the above description of $g(a, b)$ that

$$
\bar{g}(x, y)=\bigvee\{f(c, d) \mid c, d \in A \text { and } c \leq x, y \leq d\} .
$$

Thus $\overline{(B, g)}$ is, up to isomorphism, the lower MacNeille completion of $(A, f)$, and our result follows.

The following extension of Theorem 3.6 has wider applicability. It also has ties to a well-known result of Goldblatt [15] in the theory of BAOs; however, we postpone a discussion of the connection to Goldblatt's result until the following section.

Theorem 3.8. Let $K$ be a class of monotone lattice expansions of the same type, and, for some completion type $\beta$, let $\bar{K}$ be the class of $\beta$-MacNeille completions of members of $K$. If $K$ is closed under ultraproducts, then both the universal class and the variety generated by $\bar{K}$ are closed under $\beta$-canonical extensions.

Proof. Fix the completion type $\beta$. We use $\bar{L}$ for the $\beta$-MacNeille completion of $L$ and $L^{\sigma}$ for the $\beta$-canonical extension of $L$.

Let $\mathrm{S}(\bar{K})$ be the class of algebras that are isomorphic to a subalgebra of some member of $\bar{K}$. We first show that $\mathrm{S}(\bar{K})$ is closed under isomorphisms, subalgebras and ultraproducts, hence $\mathrm{S}(\bar{K})$ is the universal class generated by $\bar{K}$.

That $\mathrm{S}(\bar{K})$ is closed under isomorphisms and subalgebras follows trivially from its definition. To show $\mathrm{S}(\bar{K})$ is closed under ultraproducts suppose $L_{i} \leq \overline{M_{i}}$, where $M_{i}$ belongs to $K$ for each $i \in I$, and $\mu$ is an ultrafilter over $I$. Then by Lemma 3.7

$$
\prod_{\mu} L_{i} \leq \prod_{\mu} \overline{M_{i}} \leq \overline{\prod_{\mu} \overline{M_{i}}} \simeq \overline{\prod_{\mu} M_{i}} .
$$

As $K$ is closed under ultraproducts, $\prod_{\mu} M_{i}$ belongs to $K$, hence $\prod_{\mu} L_{i}$ belongs to $\mathrm{S}(\bar{K})$. Thus $\mathrm{S}(\bar{K})$ is indeed the universal class generated by $\bar{K}$.

We next show that $\mathrm{S}(\bar{K})$ is closed under $\beta$-canonical extensions. (We cannot apply Theorem 3.6 since we have no guarantee that $\mathrm{S}(\bar{K})$ is closed under $\beta$-MacNeille completions.) Note that by [9, Theorem 5.4], $L \leq M$ implies $L^{\sigma} \leq M^{\sigma}$. Suppose $L \leq \bar{M}$ for some $M \in K$. Then by the above remark, Theorem 3.5, and Lemma 3.7. we have

$$
L^{\sigma} \leq(\bar{M})^{\sigma} \leq \overline{(\bar{M})^{*}} \simeq \overline{\left(M^{*}\right)},
$$

where $(\bar{M})^{*}$ is a suitably saturated ultrapower $(\bar{M})^{I} / \mu$ of $\bar{M}$, and $M^{*}=M^{I} / \mu$. Thus, as $K$ is closed under ultraproducts, $M^{*}$ belongs to $K$, hence $L^{\sigma}$ belongs to $\mathrm{S}(\bar{K})$. So the universal class generated by $\bar{K}$ is closed under $\beta$-canonical extensions. 
As $\mathrm{S}(\bar{K})$ is closed under $\beta$-canonical extensions and ultraproducts, it follows from [9. Theorem 6.8] that the variety generated by $\mathrm{S}(\bar{K})$ is closed under $\beta$-canonical extensions. But the variety generated by $\mathrm{S}(\bar{K})$ is the variety generated by $\bar{K}$.

Remark 3.9. While Theorem 3.6 implies that canonical extensions are superior to MacNeille completions with respect to preservation of identities, it would be wrong to conclude that under all circumstances canonical extensions are 'better' completions than MacNeille completions. For instance, consider the class of Boolean algebras extended with two operations $\downarrow$ and $\diamond$ that preserve arbitrary existing joins. Abbreviate $\diamond^{0} x=x$ and $\diamond^{n+1} x=\diamond \diamond^{n} x$, for all $n<\omega$. Now let $C$ be the class of algebras that satisfy the following constraint:

$$
\diamond x=\bigvee_{n<\omega} \diamond^{n} x .
$$

(That is, for all $x$, the join on the right-hand side exists, and it is equal to the element on the left-hand side.) This condition plays an important role in the theory of dynamic algebras under the name of $*$-continuity; see Pratt 28 .

It is fairly easy to see that the property (3.4) is preserved under taking lower MacNeille completions, but not under taking lower canonical extensions.

Remark 3.10. For readers familiar with the notion of saturation as defined in, for instance, Chang \& Keisler [5], we briefly indicate how to use it to prove the results in this section.

For our fixed lattice $L$, let $\Sigma(L)$ be the first order signature extending the algebraic language of lattices with constants $c_{a}$ for every element $a$ of $L$, and predicate symbols $P_{F}, P_{I}$ for every filter (ideal, respectively) of $L$. Expand $L$ to a $\Sigma(L)$ structure in the obvious way. It follows from Theorems 6.1.4 and 6.1.8 in Chang \& Keisler [5] that with respect to this language, $L$ has $\kappa$-saturated elementary extensions for each cardinal $\kappa$, and that one can obtain these extensions as ultrapowers of the original structure.

In this approach, $L^{*}$ denotes an arbitrary $\omega$-saturated elementary extension of (the $\Sigma(L)$-expansion of) $L$. Then for any filter $F$ or ideal $I$, respectively, $F^{*}$ and $I^{*}$ denote the interpretation in $L^{*}$ of the monadic symbols $P_{F}$ and $P_{I}$. The same holds for $a, c_{a}$ and $a^{*}$, but we will write $i(a)$, or simply $a$, for $a^{*}$.

Let us now, as an example, see how to prove (3.1). Pick $p \in L^{*}$ and $y \in O\left(L^{\sigma}\right)$ with $p \leq \varphi(y)$, and set $I=\{a \in L \mid a \leq y\}$. Suppose for contradiction that $p \not \leq a$ for each $a \in I$. We expand the signature once more, now with the constant $p$, and obtain the signature $\Sigma(L)_{p}$; the model $L^{*}$ is expanded to a structure for this language in a natural way. Define the $\Sigma(L)_{p}$-type

$$
\Gamma(v):=\{\underline{p} \not \leq v\} \cup\left\{c_{a} \leq v \mid a \in I\right\} .
$$

For an arbitrary finite subset $\left\{a_{1}, \ldots, a_{n}\right\}$ of $I$ we find that each $a_{i}$ lies below $a_{1} \vee \cdots \vee a_{n}$, while by assumption, $p \not \leq a_{1} \vee \cdots \vee a_{n}$. From this it easily follows that $\Gamma(v)$ is finitely satisfiable in $L^{*}$, whence by 2 -saturation of $L^{*}$ there is a $z \in L^{*}$ such that $p \not \leq z$ and $a \leq z$ for all $a \in I$. By the compactness of $L$ in $L^{\sigma}$ we have $\varphi(y)=\bigvee\{i(a) \mid y \geq a \in L\}$, so $\varphi(y) \leq z$, contrary to the assumption that $p \leq \varphi(y)$. This proves (3.1).

Note that in the above proof, 2-saturation suffices because all the formulas $c_{a} \leq v$ with $a \in I$ belong to the language $\Sigma(L)$. In order to prove the other statements involving saturation, it suffices to take $L^{*}$ to be $\omega$-saturated; the price that we have 
to pay for this 'low' kind of saturation is a high cardinality of our language $\Sigma(L)$, which will generally be $2^{\kappa}$.

\section{Boolean ALgebras With OPERATORS}

Before concluding, we turn to a very special situation, viz., that of modal logic and its algebraic counterpart, Boolean algebras with operators (BAOs). We will prove three corollaries to our results, two known, and one new. All three corollaries are related to a well-known result in modal logic due to Fine [7:

The modal logic of any elementary class of Kripke frames is canonical.

Before discussing the algebraic meaning of this result, we turn to Fine's proof, which used, respectively introduced, first order and modal notions of saturation. From this proof, Goldblatt [14] extracted a result that we can see as a manifestation of Theorem 3.5 in the context of Kripke frames: it states that the so-called ultrafilter extension of a Kripke frame $S$ can be obtained as a certain 'bounded morphic image' of some elementary extension of $S$. Goldblatt [15] later generalized this result to the context of arbitrary relational structures, and showed that for the elementary extension one can actually take an ultrapower of $S$.

In order to avoid the introduction of such notions as ultrafilter extensions and bounded morphisms, we give here a more or less algebraic presentation. We do need the notion of a complex algebra.

Definition 4.1. A relational structure $S=\left(P,\left(R_{i}\right)_{i \in I}\right)$ consists of a set $P$ and a collection $\left(R_{i}\right)_{i \in I}$ of relations $R_{i}$ on $P$. A Kripke frame is a relational structure of the form $(P, R)$ with $R$ a single, binary, relation on $P$.

Given an $n+1$-ary relation $R$ on $P$, define the associated $n$-ary map $\langle R\rangle$ on the power set of $P$ by

$$
\langle R\rangle\left(Y_{1}, \ldots, Y_{n}\right)=\left\{x \in P \mid R x y_{1} \cdots y_{n} \text { for some } y_{1} \in Y_{1}, \ldots, y_{n} \in Y_{n}\right\} .
$$

Adding these maps to the power set algebra of $P$, we obtain a Boolean algebra with additional operations that we call the complex algebra of $S$ and denote by $S^{+}$.

Note that such complex algebras are rather special lattice expansions - they are complete, atomic Boolean algebras endowed with operations that are completely additive.

Goldblatt's result can now be formulated as the following corollary to Theorem 3.5

Theorem 4.2. For a relational structure $S$ there is a non-standard extension $S^{*}$ and an embedding $\varphi$ of the canonical extension of $S^{+}$in $\left(S^{*}\right)^{+}$which preserves all meets and joins.

Proof. We confine ourselves to the case that $S=(P, R)$ is a Kripke frame, and apply our results to the Boolean algebra with operators $S^{+}$. Let $\kappa$ be the cardinality of the power set of $P$, and let $\left(S^{+}\right)^{*}$ be a $\kappa^{+}$-saturated non-standard extension of $S^{+}$. It follows immediately from Theorem 3.5 that the map $\varphi$ as defined in Definition 3.1 is a complete embedding of $\left(S^{+}\right)^{\sigma}$ in the MacNeille completion of $\left(S^{+}\right)^{*}$ :

$$
\left(S^{+}\right)^{\sigma} \hookrightarrow^{c} \overline{\left(S^{+}\right)^{*}}
$$

Here we use the terms canonical extension and MacNeille completion to mean each operation is extended via its lower extension. As $\left(S^{+}\right)^{*}$ is an elementary extension 
of $S^{+}$it must also be atomic, and its atom structure will form an elementary extension $S^{*}$ of $S$. Also, since the operation $\langle R\rangle$ of $S^{+}$is completely additive, it is residuated; that is, there is a residual map $g: S^{+} \rightarrow S^{+}$such that for all $X, Y \subset P$ :

$$
\langle R\rangle X \subseteq Y \text { iff } X \subseteq g(Y) .
$$

From this it follows that $\langle R\rangle^{*}$ is residuated as well (by $g^{*}$ ), whence it preserves all existing joins. But then the lower MacNeille extension $\overline{\langle R\rangle^{*}}$ is completely additive, so that the MacNeille completion of $\left(S^{+}\right)^{*}$ is isomorphic to the complex algebra of its atom structure:

$$
\overline{\left(S^{+}\right)^{*}} \cong\left(S^{*}\right)^{+} \text {. }
$$

From the first and last displayed facts the result is immediate.

Remark 4.3. The reader may worry that this is not exactly the theorem from modal logic, since the cited modal logicians were able to obtain an extension that is $\omega$ saturated rather than the higher level of saturation required by our results. The difference can be explained by the fact that their notion of saturation is with respect to a first order language of a high cardinality - it contains a unary predicate symbol for every subset of the Kripke frame $S$. Had we chosen to work along similar lines (as sketched in Remark 3.10), then we would have obtained a version of our results referring to $\omega$-saturated extensions as well.

Remark 4.4. Instead of viewing Theorem 4.2 as an application of our results, one may think of Theorem 3.5 as a version of the modal result which is applicable in a much more general setting. This may be useful when treating logics, such as substructural logics of various kinds, whose algebraic counterparts are not classes of BAOs but classes of possibly non-distributive lattice expansions.

To explain the meaning of (4.1) in more algebraic terms it is convenient to introduce some terminology.

Definition 4.5. Given a class $K$ of relational structures, $V(K)$ denotes the variety of algebras generated by the complex algebras of structures in $K$. That is, $V(K)=$ HSPCm $(K)$, where $\mathrm{Cm}$ denotes the class operation of taking complex algebras.

Definition 4.6. A variety of BAOs is said to be canonical if it is closed under lower canonical extensions.

Fine's result (4.1) then translates as follows: An elementary class of Kripke frames generates a canonical variety of modal algebras. We remind the reader that a Kripke frame is a relational structure with a single binary relation (see Definition 4.1). In subsequent years this theorem has been strengthened and generalized; for instance, Goldblatt [15] proves Theorem 4.7 below. We can now provide a purely algebraic proof of this result, as a corollary to Theorem 3.8

Theorem 4.7. If $K$ is a class of relational structures that is closed under ultraproducts, then the variety $V(K)$ is canonical.

Proof. Let $C=\operatorname{PuCm}(K)$ be the class of algebras obtained as ultraproducts of complex algebras of members of $K$. We claim $\bar{C}=\mathrm{Cm}(K)$. Indeed, if $A \in \mathrm{Cm}(K)$, then $A \in C$ and $A$ is complete, hence $A=\bar{A} \in \bar{C}$. Conversely, if $A \in \bar{C}$, then $A=\overline{\prod_{\mu} S_{i}^{+}}$for some family $S_{i}(i \in I)$ of structures in $K$ and some ultrafilter $\mu$ over $I$. Then, as in the proof of Theorem 4.2, $\overline{\prod_{\mu} S_{i}^{+}}$is isomorphic to $\left(\prod_{\mu} S_{i}\right)^{+}$, 
and as $K$ is closed under ultraproducts, it follows that $A$ belongs to $\mathrm{Cm}(K)$. By construction, $C$ is closed under ultraproducts. Therefore, by Theorem $3.8, V(\bar{C})$ is canonical, and so the theorem follows, as $\bar{C}=\mathrm{Cm}(K)$.

The final application of our results concerns the converse of Fine's theorem, rather than its proof. It has long been an open problem in the theory of BAOs whether this converse holds; that is, whether every canonical variety of BAOs is generated by some class of relational structures that is closed under ultraproducts; or, equivalently (as shown by Goldblatt [16]), whether every canonical variety of BAOs is generated by some elementary class of relational structures.

Goldblatt, Hodkinson \& Venema 18, have recently shown this is not the case, however, as they point out, it remains an outstanding problem to characterize those varieties of BAOs that are generated by such a class of structures. There is a list of positive answers to this question in [18; one of these results involves the notion of singleton persistence, also known as di-persistence. We need the following definition.

Definition 4.8. Let $S$ be a relational structure; its singleton algebra $S^{\circ}$ is the subalgebra of $S^{+}$generated by the atoms of $S^{+}$(i.e., by the singletons of $S$ ).

Given a class $C$ of Boolean algebras with operators, $S$ is called a structure for $C$ if $S^{+}$belongs to $C$, and a weak structure for $C$ if $S^{\circ}$ belongs to $C$. The class of structures and of weak structures for $C$ are denoted as $\operatorname{Str}(C)$ and $\operatorname{Wst}(C)$, respectively.

The relevance of these notions is based on the following two facts. First, it is well known and easy to see that any canonical variety is generated by its structures: for such $V$ it even holds that $V=\operatorname{SCmStr}(V)$. Second, it has been shown by Venema [32] that for any universal class $C$ of BAOs, the class Wst $(C)$ is elementary. In fact, first order formulas defining Wst $(C)$ can be effectively obtained from the universal formulas characterizing $C$. Put together, these facts explain the interest in cases where the classes $\mathrm{Wst}(V)$ and $\operatorname{Str}(V)$ coincide.

Definition 4.9. A variety $V$ of BAOs is singleton persistent if $\operatorname{Wst}(V)=\operatorname{Str}(V)$.

Goldblatt [17] proves that every variety which is both singleton persistent and canonical is generated by an elementary class of relational structures. Our contribution here is that both conditions, that is, canonicity and singleton persistence, follow from the variety being closed under taking MacNeille completions.

Theorem 4.10. Let $V$ be a variety of Boolean algebras with operators that is closed under lower MacNeille completions. Then $V$ is singleton persistent and canonical. Hence $V$ is generated by an elementary class of relational structures, namely $\operatorname{Str}(V)$.

Proof. One easily shows that the complex algebra of any relational structure $S$ is the lower MacNeille completion of its singleton algebra: $S^{+}=\overline{S^{\circ}}$. From this observation and the assumption on $V$ it follows that $\operatorname{Wst}(V) \subseteq \operatorname{Str}(V)$. Since the opposite inclusion holds for any class of algebras that is closed under taking subalgebras, we find that $\mathrm{Wst}(V)=\operatorname{Str}(V)$. That is, $V$ is singleton persistent. (This is essentially Theorem 5.1(a) of Goldblatt [17); our contribution here has been to remove the restriction to complete additivity.) Theorem 3.6 gives directly that $V$ is canonical. The last part of the theorem then follows from Corollary 3.4 of Goldblatt [17] — or from the two facts given above Definition 4.9. 


\section{REFERENCES}

[1] B. Banaschewski, Hüllensysteme und Erweiterungen von Quasi-Ordnungen, Z. Math. Logik Grundl. Math. 2 (1956), 35-46. MR0082447(18,551a)

[2] D.R. Balbes and P. Dwinger, Distributive Lattices, University of Missouri Press, 1974. MR0373985 (51:10185)

[3] P. Blackburn, M. de Rijke and Y. Venema, Modal Logic, Cambridge University Press, 2001. MR.1837791 (2003b:03001)

[4] S. Burris and H.P. Sankappanavar, A Course in Universal Algebra, Springer, 1981. MR0648287 (83k:08001)

[5] C.C. Chang and H.J. Keisler, Model Theory, Stud. Logic Found. Math., Vol. 73, NorthHolland, Amsterdam, 1973. MR0409165 (53:12927)

[6] P. Crawley and R.P. Dilworth, Algebraic Theory of Lattices, Prentice-Hall, Englewood Cliffs, New Jersey, 1973.

[7] K. Fine, Some connections between elementary and modal logic, in [24], pp. 15-31. MR0401437 (53:5265)

[8] M. Gehrke, Robinson lattices and their spectra, Algebra Universalis 32 (1994), 204-223. MR.1290159 (96e:06009)

[9] M. Gehrke and J. Harding, Bounded lattice expansions, J. Algebra 238 (2001), 345-371. MR1822196 (2002d:06005)

[10] M. Gehrke and B. Jónsson, Bounded distributive lattice expansions, Math. Scand. 94 (2004), 13-45. MR 2032334 (2004j:06008)

[11] M. Gehrke, H. Nagahashi, and Y. Venema, A Sahlqvist theorem for distributive modal logic, Ann. Pure Applied Logic, to appear. (Also available as Technical report, Institute for Logic, Language and Computation, University of Amsterdam, 2002.)

[12] G. Gierz, K.H. Hoffmann, K. Keimel, J.D. Lawson, M. Mislove and D.S. Scott, A Compendium of Continuous Lattices, Springer Verlag, 1980. (Second edition, Cambridge University Press, 2003.)

[13] S. Givant and Y. Venema, The preservation of Sahlqvist equations in completions of Boolean algebras with operators, Algebra Universalis 41 (1999), 47-84. MR.1682042 (2000e:06019)

[14] R. Goldblatt, Metamathematics of modal logic, Reports on Mathematical Logic 6 (1976), 41-78, and 7 (1976) 21-52. MR0536322 (58:27331b)

[15] R. Goldblatt, Varieties of complex algebras, Ann. Pure Applied Logic 44 (1989), 173-242. MR.1020344 (91d:08005)

[16] R. Goldblatt, Elementary Generation and Canonicity for Varieties of Boolean Algebras with Operators, Algebra Universalis 34 (1995), 551-607. MR1357484 (97a:06028)

[17] R. Goldblatt, Persistence and atomic generation for varieties of Boolean algebras with operators, Studia Logica 68 (2001), 155-171. MR1860730 (2002g:08013)

[18] R. Goldblatt, I. Hodkinson and Y. Venema, Erdös graphs resolve Fine's canonicity problem, Bulletin of Symbolic Logic 10 (2004), 186-208. MR2062417

[19] R. Goldblatt, Mathematical Modal Logic: a View of its Evolution, Journal of Applied Logic, 1 (2003), 309-392. MR 2021314 (2004i:03031)

[20] J. Harding, Canonical completions of lattices and ortholattices, Tatra Mt. Math. Publ. 15 (1998), 85-96. MR1655081 (2000a:06033)

[21] R. Hirsch and I. Hodkinson, Relation Algebras by Games, Stud. Logic Found. Math., Vol. 147, North-Holland, Amsterdam, 2002. MR 1935083 (2003m:03001)

[22] B. Jónsson and A. Tarski, Boolean algebras with operators I, Amer. J. Math. 73 (1951), 891-993. MR0044502 (13:426c)

[23] B. Jónsson and A. Tarski, Boolean algebras with operators II, Amer. J. Math. 74 (1952), 127-162. MR0045086 (13:524g)

[24] S. Kanger (editor), Proceeding of the Third Scandinavian Logic Symposium (Univ. Uppsala, Uppsala, 1973), Stud. Logic Found. Math., Vol. 82, North-Holland, Amsterdam, 1975. MR0369020(51:5256)

[25] M.D. MacLaren, Atomic orthocomplemented lattices, Pacific J. Math. 14 (1964), 597-612. MR0163860 (29:1159)

[26] H.M. MacNeille, Partially ordered sets, Trans. AMS 42 (1937), 416-460. MR1501929

[27] J.D. Monk, Completions of Boolean algebras with operators, Math. Nachr. 46 (1970), 47-55. MR0277369(43:3102) 
[28] V. Pratt, Dynamic algebras: examples, constructions, applications, Studia Logica 50 (1991), 571-601. MR:1170187 (93h:03086)

[29] H. Sahlqvist, Completeness and correspondence in the first and second order semantics for modal logic, in [24], pp. 110-143. MR0387008 (52:7855)

[30] J. Schmidt, Zur Kennzeichnung der Dedekind-MacNeilleschen Hülle einer geordneten Menge, Archiv. d. Math. 7 (1956), 241-249. MR0084484 (18,868a)

[31] K.D. Stroyan and W.A.J. Luxemburg, Introduction to the Theory of Infinitesimals, Academic Press, 1976. MR0491163 (58:10429)

[32] Y. Venema, Atom structures, in: M. Kracht et alii (eds.), Advances in Modal Logic, Volume 1, CSLI Publications, Stanford, 1997, pp. 291-305. MR1688528

Department of Mathematical Sciences, New Mexico State University, Las Cruces, New Mexico 88003

E-mail address: mgehrke@nmsu.edu

$U R L:$ http://www . math.nmsu.edu/mgehrke/mgehrke.html

Department of Mathematical Sciences, New Mexico State University, Las Cruces, New MeXico 88003

E-mail address: jharding@nmsu.edu

$U R L:$ http://math.NMSU.Edu/JohnHarding.html

Institute for Logic, Language and Computation, University of Amsterdam, Plantage Muidergracht 24, 1018 TV Amsterdam, Netherlands

E-mail address: yde@science.uva.nl

$U R L:$ http://staff.science.uva.nl/〜yde/ 